\title{
SPLICS: a split green fluorescent protein-based contact site sensor for narrow and wide heterotypic organelle juxtaposition
}

\author{
Domenico Cieri ${ }^{1}$ - Mattia Vicario ${ }^{1}$ - Marta Giacomello ${ }^{2,3} \cdot$ Francesca Vallese $^{1} \cdot$ Riccardo Filadi $^{1} \cdot$ Tina Wagner $^{2,3}$. \\ Tullio Pozzan ${ }^{1,4,5} \cdot$ Paola Pizzo ${ }^{1,5} \cdot$ Luca Scorrano $^{2,3} \cdot$ Marisa Brini $^{2} \cdot$ Tito Cali $^{1}$
}

Received: 1 July 2017 / Revised: 17 October 2017 / Accepted: 31 October 2017

(c) The Author(s) 2018. This article is published with open access

\begin{abstract}
Contact sites are discrete areas of organelle proximity that coordinate essential physiological processes across membranes, including $\mathrm{Ca}^{2+}$ signaling, lipid biosynthesis, apoptosis, and autophagy. However, tools to easily image inter-organelle proximity over a range of distances in living cells and in vivo are lacking. Here we report a split-GFP-based contact site sensor (SPLICS) engineered to fluoresce when organelles are in proximity. Two SPLICS versions efficiently measured narrow $(8-10 \mathrm{~nm})$ and wide $(40-50 \mathrm{~nm})$ juxtapositions between endoplasmic reticulum and mitochondria, documenting the existence of at least two types of contact sites in human cells. Narrow and wide ER-mitochondria contact sites responded differently to starvation, ER stress, mitochondrial shape modifications, and changes in the levels of modulators of ER-mitochondria juxtaposition. SPLICS detected contact sites in soma and axons of D. rerio Rohon Beard (RB) sensory neurons in vivo, extending its use to analyses of organelle juxtaposition in the whole animal.
\end{abstract}

\section{Introduction}

In eukaryotic cells, organelles are often found in close proximity, leading to the generation of heterotypic membrane appositions that ensure the coordination of several

Edited by N. Chandel

These authors contributed equally: Domenico Cieri, Mattia Vicario and Marta Giacomello.

Electronic supplementary material The online version of this article (https://doi.org/10.1038/s41418-017-0033-z) contains supplementary material, which is available to authorized users.

Marisa Brini

marisa.brini@unipd.it

$\triangle$ Tito Calì

tito.cali@unipd.it

1 Department of Biomedical Sciences, University of Padova, Padova, Italy

2 Department of Biology, University of Padova, Padova, Italy

3 Dulbecco-Telethon Institute, Venetian Institute of Molecular Medicine, Padua, Italy

4 Venetian Institute of Molecular Medicine, Padua, Italy

5 Department of Biomedical Sciences, Institute of Neuroscience, Italian National Research Council (CNR), Padua, Italy cellular activities. Indeed, a network of contact sites between membranes of different organelles guarantees their mutual communication by creating microdomains that favor different signaling and metabolic pathways $[1,2]$. Due to their central role in many fundamental cell processes, the sites of apposition between mitochondria and the endoplasmic reticulum (ER), which range from 10 to $100 \mathrm{~nm}$, are, so far, the best characterized [3-5].

Several approaches are currently available to assess ER-mitochondria contact sites. Electron microscopy (EM) allows to calculate contact site distance, but it is timeconsuming. The in situ proximity ligation assay is based on the use of pairs of primary antibodies against proteins on opposing membranes [6]. It is widely used [7-9] but is not devoid of drawbacks: as the EM, it can only be used in fixed cells and is limited by the availability and the specificity of the antibodies.

The use of fluorescent proteins (FPs) selectively targeted to the mitochondrial matrix and the lumen of the ER [10] has been the golden standard to visualize contact sites in living cells for years. However, limited resolution in the distance range below $200 \mathrm{~nm}$, differences in FPs expression levels or alterations in organelle morphology complicated the interpretation of experiments of ER-mitochondria juxtaposition upon ablation of the mitochondria-shaping protein Mitofusin 2 (Mfn2) [11-13]. 
To overcome these limits, FP-based sensors of proximity were developed: a dimerization-dependent FP (ddGFP) [14] or Venus FP $[2,15,16]$ and a FRET-based probe coupled to a rapamycin-binding module (FEMP) [17]. While these two probes improved the analysis of ER-mitochondria proximity, they also face some limitations: the ddGFP probe is intrinsically not extremely bright [14]; the FRET probe requires equimolar expression of the two moieties [12] and its in vivo applications are limited by the use of rapamycin, a potent inducer of autophagy [18-20], to maximize juxtaposition and FRET signal. Moreover, both probes cannot be adapted for the investigation of contact sites potentially placed at different distances, because their dynamic range must be characterized each time that the linker is changed. Artificial GFP-based tethers have proved useful to uncover a novel ER-mitochondria tethering complex in yeast, but they cannot been used to monitor changes in the ER-mitochondria contact sites [21]. Therefore, an easy, one-step probe that can dynamically detect ER-mitochondria juxtaposition in cellulo and in vivo is lacking.

To overcome these limitations, we devised a split-GFPbased contact site sensor (SPLICS) that can be easily adapted to measure ER-mitochondria contact sites over a range of distances as well as other types of hetero and homotypic contact sites. Upon expression in human cells, this one-step imaging technique specifically identifies narrow and wide ER-mitochondria apposition lying in a range of around 10 and $50 \mathrm{~nm}$ [5], i.e, that found between mitochondria and smooth or rough ER [22]. The narrow SPLICS can also detect ER-mitochondria contact sites in vivo in zebrafish sensory neurons. Pharmacological and genetic manipulations indicate that these narrow and wide contact sites respond differentially to ER stress, autophagy, mitophagy, and changes in the levels of modulators of ER-mitochondria juxtaposition.

In conclusion, using SPLICS as a tool to investigate ER-mitochondria contact sites, we unravel their heterogeneity and provide the community with a sensor that can be easily adapted to image other types of heterotypic organelle contact sites in human cells and in whole organisms.

\section{Results}

\section{Two SPLICS probes for different ranges of ER-mitochondria juxtaposition}

To generate a modular fluorescence based sensor of organelle proximity, we decided to capitalize on the ability of two nonfluorescent portions (the $\mathrm{GFP}_{1-10}$ moiety and the GFP $\beta$-strand 11) of the superfolder GFP variant [23-25] to restore a fully fluorescent GFP upon self-assembly. We reasoned that if we targeted each moiety on one of the juxtaposed membranes, the GFP fluorescence would be restored only when the two portions were close enough. We therefore placed the nonfluorescent $\mathrm{GFP}_{1-10}$ moiety on the cytosolic face of the OMM $\left(\mathrm{OMM}-\mathrm{GFP}_{1-10}\right)$. To follow short- $(\approx 8-10 \mathrm{~nm})$ and longrange $(\approx 40-50 \mathrm{~nm})$ ER-mitochondria interactions [5], two constructs that differ for the length of the spacer placed between the ER targeting sequence and the $\beta_{11}$ fragment were created by considering the distance of $0.36 \mathrm{~nm}$ between two alpha-carbons in a peptide chain: a ER-Short $\beta_{11}$ with a 29 aa spacer and a ER-Long $\beta_{11}$ with a 146 aa spacer (i.e., a maximum of $\approx 10.4$ and $52.5 \mathrm{~nm}$, respectively). These values might clearly be subjected to changes (i.e., reduction) since the amino acid sequences might not always be fully extended. We reasoned that co-expression of ER-Short $\beta_{11}$ with OMM$\mathrm{GFP}_{1-10}\left(\mathrm{SPLICS}_{\mathrm{S}}\right)$ and of ER-Long $\beta_{11}$ with OMM-GFP ${ }_{1-10}$ $\left(\right.$ SPLICS $\left._{\mathrm{L}}\right)$ would result in reconstitution of GFP fluorescence (Fig. 1a). Two additional constructs, a $\beta_{11}$-tagged FP (Kate$\beta_{11}$ ) and an untargeted $\mathrm{GFP}_{1-10}$, were also generated to verify the complementation of the OMM-GFP ${ }_{1-10}$ at the OMM (Fig. 1a, left) and the $\mathrm{ER}_{\mathrm{S} / \mathrm{L}}-\beta_{11}$ at the ER (Fig. 1a, middle), respectively. Expression of SPLICS $S_{S}$ and SPLICS $_{L}$ will result in fluorescence emission specifically at the ER-mitochondria interface (Fig. 1a, right).

The different versions of the SPLICS probes were first tested for their correct localization and topology. A clear mitochondrial network appeared in HeLa cells coexpressing OMM-GFP ${ }_{1-10}$ with Kate- $\beta_{11}$ (Fig. $1 b$, first panels); similarly, the ER network became fluorescent when $E R_{S^{-}} \beta_{11}$ and $E_{L^{-}} \beta_{11}$ were co-expressed with a cytosolic non-fluorescent $\mathrm{GFP}_{1-10}$ (Fig. 1b, second and third panel couples). Interestingly, when SPLICS or $_{\text {SPLICS }}$ were expressed in HeLa (Fig. 1b, fourth and fifth panel couples) and in HEK293 cells (Supplementary Figure S1), fluorescent individual foci appeared, likely representing the juxtapositions between ER and mitochondria. At a closer inspection, the SPLICS $S_{S}$ and SPLICS $_{\mathrm{L}}$ signals retrieved in HeLa cells appeared different for number (see quantification in 3D rendered z-stack images, Fig. 1c).

We therefore verified whether SPLICS really recognized areas of ER-mitochondria juxtaposition. In HeLa cells

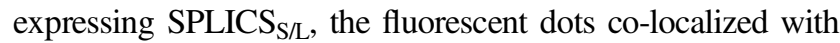
endogenous markers of mitochondria (mtHSP60) and ER (calreticulin) (Fig. 1d). Noteworthy, the mitochondrial and ER networks were not completely engaged in the formation of the ER-mitochondria contacts reported by the SPLICS (see merge panels of Fig. 1d), suggesting that SPLICS snapshots the juxtaposition at any given moment even when transiently formed. Immuno-EM with anti-GFP antibody revealed that mitochondria and ER membranes in contact with mitochondria were preferentially marked (arrowheads in Supplementary Figure S2). Despite the non-complemented and complemented OMM-GFP ${ }_{1-10}$ cannot be distinguished by the 
characterization of the SPLICS probes. a Cartoon showing the general approach used to design the SPLICS. The mitochondrial network, the ER network, and the ER-mitochondria contact sites are revealed by coexpression of the $\beta_{11}$-tagged cytosolic RFP (Kate) and the OMM-GFP $_{1-10}$ (left panel), of the $E R_{S / L}-\beta_{11}$ constructs and a cytosolic GFP $_{1-10}$ (middle panel) and of the SPLICS $\mathrm{S}_{\mathrm{S} / \mathrm{L}}$ (right panel), respectively. $\mathbf{b}$

Experimental controls showing the correct targeting of the mitochondrial (OMM-GFP ${ }_{1-10}$ ) and the ER $\left(\mathrm{ER}_{\mathrm{S}^{-}} \beta_{11}\right.$ and $\mathrm{ER}_{\mathrm{L}^{-}}$ $\beta_{11}$ ) targeted fragments verified by complementation with Kate$\beta_{11}$ and $\mathrm{GFP}_{1-10}$, respectively. Co-transfection of HeLa cells with OMM-GFP $1-10$ and both $\mathrm{ER}_{\mathrm{S}}-\beta_{11}$ or $\mathrm{ER}_{\mathrm{L}}-\beta_{11}$ induces the appearance of a "dotted" fluorescence. c Quantification of ER-mitochondria contacts in HeLa cells. The SPLICS dots were quantified from the $3 \mathrm{D}$ rendering of a complete $\mathrm{z}$-stack. Mean \pm SEM: SPLICS $_{\mathrm{S}} 56 \pm 4$, $n=37$ cells; SPLICS $_{\mathrm{L}} 229 \pm 12$, $n=25$ cells. d Co-localization

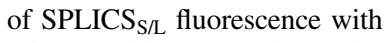
mitochondria (mtHSP60) and ER (CRT: calreticulin) markers. Representative traces $\mathbf{e}$ and statistical analysis $\mathbf{f}$ of mitochondrial $\mathrm{Ca}^{2+}$ uptake in HeLa cells transfected with mtAeqmut. Mean \pm SEM: Void Vector $75 \pm 2, n=65$ wells; SPLICS $_{\mathrm{S}} 77 \pm 1, n=54$ wells; SPLICS $_{\mathrm{L}} 71 \pm 2, n=54$ wells. Scale bar $15 \mu \mathrm{m}$. Data shown are the result of 3-5 independent experiments
Fig. 1 Functional

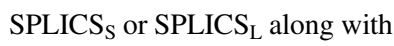
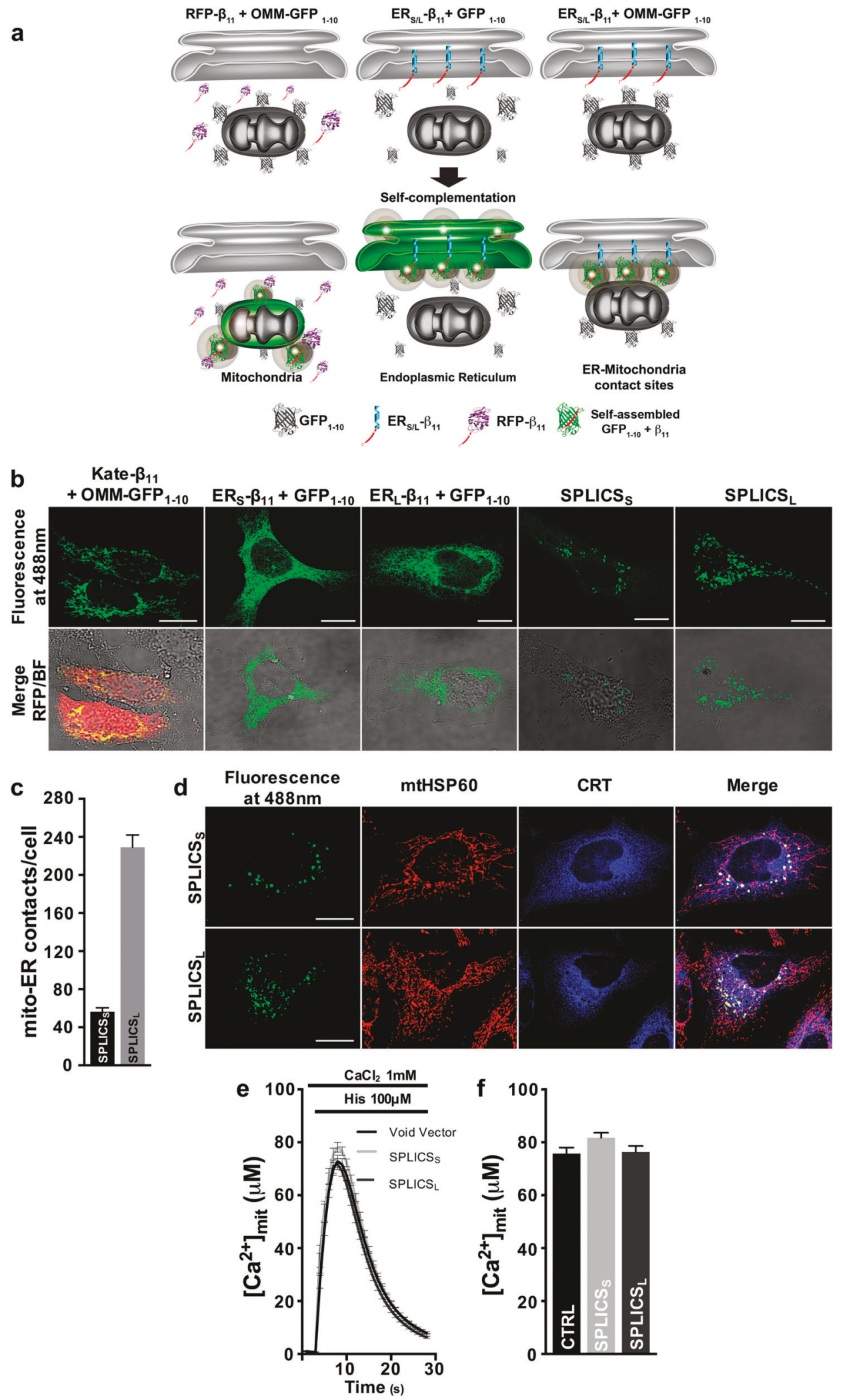

anti-GFP antibody, it is evident that a consistent number of gold nanoparticles are distributed at the ER-mitochondria interface (inset in Supplementary Figure S2).
To gain further insights on the nature of the reconstituted SPLICs, we evaluated their stability by checking whether the number of SPLICS $S_{S / L}$ foci could change after 24,48 , 
and $72 \mathrm{~h}$ post transfection. Supplementary Figure S3 shows that the number of SPLICS $_{\mathrm{S} / \mathrm{L}}$ is stable during the time course. The number of fluorescent reconstituted foci was also unaffected by the expression level of the probes (Supplementary Figure S4), suggesting that bona fide changes in the SPLICS $S_{S / L}$ fluorescent foci likely reflect a variation in ER-mitochondria contact sites number rather than differences in the stability/expression levels of the probes. Additionally, the overall morphology of the ER and mitochondria in cells expressing the SPLICS $S_{S / L}$ remained grossly unaltered (Supplementary Figure S5).

To exclude that novel and non-physiological contact sites between ER and mitochondria might be artificially induced by SPLICS expression, ER-mitochondria $\mathrm{Ca}^{2+}$ transfer and mitochondrial $\mathrm{Ca}^{2+}$ uptake were evaluated in HeLa cells expressing SPLICS $S_{S / L}$ by aequorin-based measurements. If this was the case, mitochondrial $\mathrm{Ca}^{2+}$ transients generated by stimulation with the $\mathrm{InsP}_{3}$-linked agonist histamine should be increased in SPLICS-expressing cells [17]; however, they were superimposable to those of control cells (Fig. If and quantification in Fig. 1g). Taken together, these results indicate that SPLICS retains the ability to selfassociate only in specific areas where the two organelles are found within the distance imposed by the linker region and that it does not artificially increase tethering and $\mathrm{Ca}^{2+}$ transfer between ER and mitochondria.

\section{Modulation of short- and long-range ER-mitochondria interfaces during ER stress and autophagy}

We next wished to address if SPLICS could respond to pathophysiological conditions known to affect the extent of ER-mitochondria contacts. We therefore measured shortand long-range ER-mitochondria interactions in conditions where increased ER-mitochondria coupling was reported, such as ER stress and induction of autophagy [5, 26, 27]. In HeLa cells treated with the ER stress inducer tunicamycin, or starved, the number of short-range ER-mitochondria contact sites measured by SPLICS $_{\mathrm{S}}$ were increased (Fig. 2a, b), in agreement with previous results [5, 26, 27]. The picture in the case of long-range ER-mitochondria interactions measured by the SPLICS $_{\mathrm{L}}$ was more complex: while tunicamycin significantly decreased the number of SPLICS dots, starvation did not induce any significant change (Fig. 2c, d). Altogether, these results indicate that short and long ER-mitochondria interactions are differentially modulated in response to different stimuli and
Fig. 2 Effect of Tunicamycin and Hbss treatment on ER-mitochondria contacts. Immunofluorescence against mitochondria (Tom20, red) is shown in the panels on the middle. The green channel is the merge of several planes. Scale bar $20 \mu \mathrm{m}$. a Representative confocal pictures of HeLa cells expressing the SPLICS $_{\mathrm{S}}$ probe. b Quantification of SPLICS contacts by $3 \mathrm{D}$ rendering of complete z-stacks. Mean \pm SEM: Ctrl $58 \pm 3, n=32$ cells; Tunicamycin $84 \pm 5, n=33$ cells; Hbss $81 \pm 5, n=25$ cells. c Representative confocal pictures of HeLa cells expressing the SPLICS $_{\mathrm{L}}$ probe. d Quantification of SPLICS contacts by $3 \mathrm{D}$ rendering of complete z-stacks. Mean \pm SEM: Ctrl $218 \pm 11, n=27$ cells; Tunicamycin $171 \pm 9, n=$ 33 cells; Hbss $204 \pm 10, n=23$ cells. Data shown are the result of three independent experiments. $* * p \leq 0.01, * * * p \leq$ 0.001

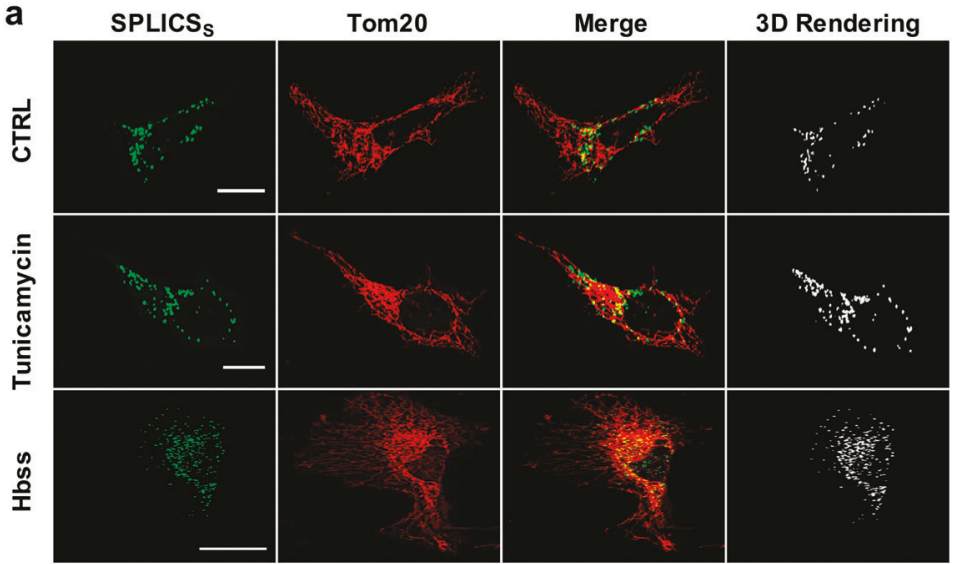

b

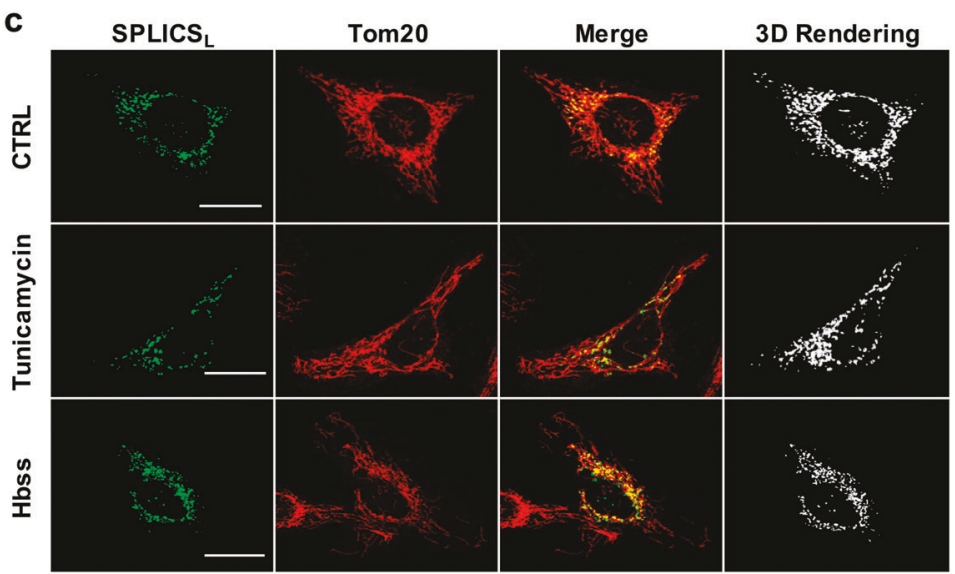

d

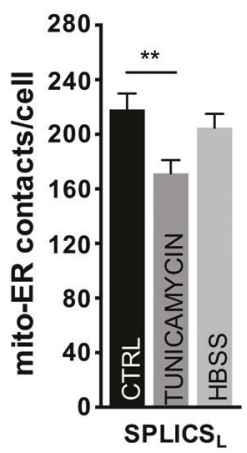


suggest that the heterogeneity between the two types of contact sites reflects their involvement in specialized cellular pathways.

\section{Short- and long-range ER-mitochondria interactions are differentially modulated by mitochondrial morphology}

During starvation, inhibition of the mitochondrial fission GTPase Dynamin-related protein 1 (Drp1) results in mitochondrial elongation, increasing energy conversion and sparing mitochondria from autophagosomal degradation $[28,29]$. We therefore wished to verify short and long ER-mitochondria interactions upon Drp1-driven mitochondrial shape changes. We expressed wt or a dominantnegative mutant form of Drp1 (Drp1-K38A) to induce mitochondrial fragmentation or elongation and measured the occurrence of short- and long-range ER-mitochondria juxtaposition with SPLICS. Mitochondrial fragmentation induced by wt Drp1 expression did not change the number of short-range ER-mitochondria interactions (Fig. 3a and b, compare top panels vs. middle panels), in agreement with previous data [30]. Conversely, mitochondrial elongation induced by dominant-negative Drp1 expression resulted in a significant increase in the short-range ER-mitochondria contacts detected by SPLICS $_{\mathrm{S}}$ (Fig. 3a, top panels vs. lower panel, and Fig. 3b). The SPLICS ${ }_{\mathrm{L}}$ measured a significant reduction in the number of wide ER-mitochondria interactions in cells expressing wt Drp1 (Fig. 3c top panels vs middle panels, and Fig. 3d). Interestingly, forced mitochondrial elongation induced by Drp1-K38A expression resulted in the labeling of the whole surface of mitochondria by SPLICS $_{\mathrm{L}}$ fluorescence, suggesting a complete engagement of the mitochondrial network with the ER (Fig. 3c, top panels vs. bottom panels). Due to the filamentous nature of the observed SPLICS $_{\mathrm{L}}$ staining, the number of ER-mitochondria contacts/cell under this condition could not be reliably quantified; nevertheless, the GFP signal occupied almost completely (about $85 \%$ ) the mitochondrial surface as measured by Tom 20 staining (Supplementary Figure S6). Altogether, these results suggest that unopposed mitochondrial fusion is paralleled by an enhancement of the ER-mitochondria interface that may ensure the supply of lipids required for the sustained mitochondrial morphological changes [26, 28, 29, 31].

\section{Short- and long-range ER-mitochondria contacts respond differentially to $M$ fn2 2 silencing and presenilin 2 mutant expression}

We next wished to verify if SPLICS responded to genetic modulation of the ER/mitochondria interaction. To this end, we decided to monitor SPLICS $_{\mathrm{S} / \mathrm{L}}$ behavior following ablation of Mitofusin 2 (Mfn2), a pro-fusion mitochondria-
Fig. 3 Effects of Drp1

overexpression on

ER-mitochondria contacts.

Immunofluorescence against mitochondria (Tom20, cyan) and Drp1 (red) is shown in the corresponding panels. The green channel is the merge of several planes. Scale bar $20 \mu \mathrm{m}$. a Representative confocal pictures of HeLa cells expressing the SPLICS $_{S}$ probe. b

Quantification of SPLICS contacts by $3 \mathrm{D}$ rendering of complete z-stacks. Mean \pm SEM: Ctrl $59 \pm 3, n=79$ cells; Drp1 WT $70 \pm 5, n=32$ cells; Drp1-K38A $89 \pm 9, n=28$ cells. c Representative confocal pictures of HeLa cells expressing the SPLICS $_{\mathrm{L}}$ probe. d Quantification of SPLICS contacts by $3 \mathrm{D}$ rendering of complete z-stacks. Mean \pm SEM: Ctrl $260 \pm 14, n=24$ cells; Drp1 WT $198 \pm 14, n=$ 24 cells. Data shown are the result of 3-4 independent experiments. $* * p \leq 0.01$
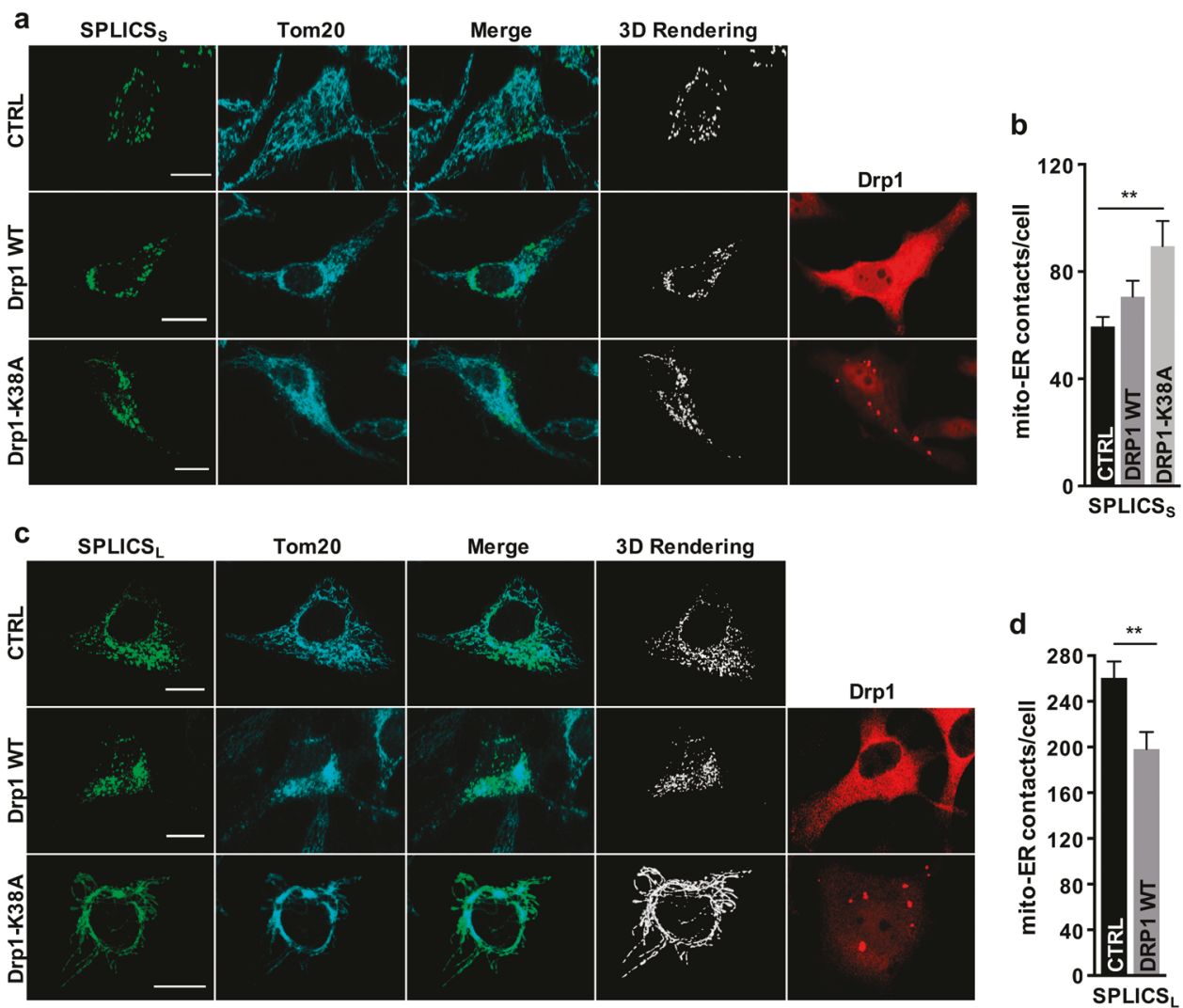

3D Rendering
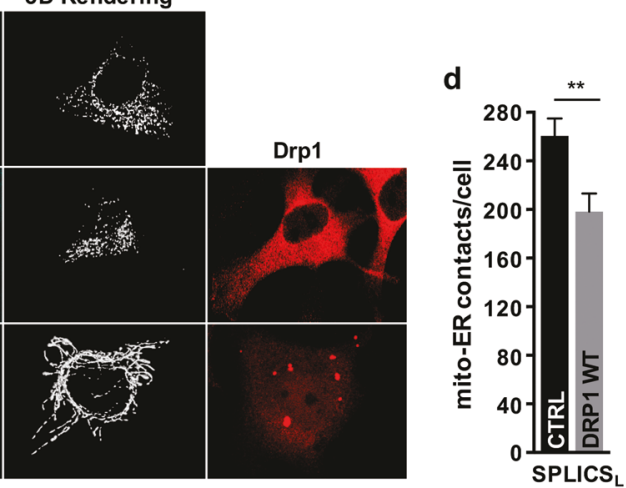
shaping protein originally identified as a tether between the two organelles [11]. However, whether Mfn2 tethers $[12,14,32-35]$ or separates [13, 22, 36-38] ER and mitochondria is still a matter of debate. We reasoned that SPLICS $_{\mathrm{S} / \mathrm{L}}$ might contribute to clarify the issue by providing an estimate of the contact sites over different ranges of interaction. Acute downregulation of Mfn2 by shRNA in HeLa cells by three independent shRNA (Supplementary Figure S7) increased by $\approx 40 \%$ the number of SPLICS $_{S}$ foci (Fig. 4a, b). Conversely, under the same conditions of Mfn2 downregulation the SPLICS ${ }_{\mathrm{L}}$ detected a significant decrease by $\approx 30 \%$ in the number of ER-mitochondria interactions (Fig. 4c, d). Altogether the short- and long-range SPLICS probes not only respond to changes in known modulators of ER-mitochondria tethering, but they might also prove useful to shed light on the observed discrepancies on the role of Mfn2 at the ER-mitochondria interface.

Mfn2 and the Familial Alzheimer's Disease (FAD)related protein Presenilin-2 (PS2) have been reported to act in a common route to tune the ER-mitochondria interface $[38,39]$. We measured short-range ER-mitochondria interactions in human fibroblasts from an FAD-patient carrying the PS2-N141I mutation, previously shown to enhance ER-mitochondria coupling in an Mfn2-dependent manner [38], and a healthy sex- and age-matched control. The SPLICS $_{S}$ signal was more than doubled in human FAD-PS2 fibroblasts compared to controls, thus confirming that endogenous FAD-PS2 increases ER-mitochondria coupling, as already reported, and proving that SPLICS represents a useful tool also in patient-derived samples (Fig. 4e, f). Lastly, we tested SPLICS $S_{S / L}$ with an additional well-established tethering machinery, i.e., the VAPB/ PTPIP51 complex. Interestingly, we detected an increase

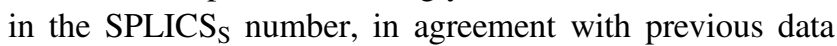
[7, 8, 40] (Supplementary Figure S8). The long-range interactions monitored by SPLICS ${ }_{L}$ were instead decreased (Supplementary Figure S8): this finding certainly deserves additional experiments but again, it might indicate that ER-mitochondria tethering can be heterogeneous and tightly modulated.

\section{Long- and short-range ER mitochondria contacts reduction during Parkin-mediated mitophagy}

Comforted by the ability of SPLICS $S_{S / L}$ to provide insights under pharmacological and genetic manipulation of the ER-mitochondria interface, we decided to detect changes in ER-mitochondria tethering during Parkin-mediated mitophagy. In mammalian cells, dysfunctional mitochondria recruit the E3 ubiquitin ligase Parkin to the OMM through PINK1 kinase activity, resulting in the recruitment and activation of the autophagy machinery [41]. Parkin has been shown to act as a positive modulator of ER and mitochondria coupling in HeLa cells by organelle-targeted FPs, and in nigral neurons by transmission EM analysis [42, 43]. Nevertheless, increased ER-mitochondria juxtaposition in patient-derived fibroblasts and in PARK2 knockout MEFs [44] was also reported. Thus, the exact function of Parkin at the ER-mitochondria interface under basal conditions and upon mitophagy is unclear. We generated a bicistronic vector in which Parkin was cloned upstream of a self-cleaving viral 2A peptide (P2A) [45] followed by a plasma membrane-targeted RFP (mCherryCAAX) to track Parkin-positive cells (Supplementary Figure S9). This construct was co-expressed along with SPLICS $_{\mathrm{S} / \mathrm{L}}$ in HeLa cells where Parkin is absent or weakly expressed [46, 47]. Parkin overexpression increased SPLICS $_{S}$ number (Fig. 5a, b), in agreement with our previous data [42]. Conversely, Parkin overexpression reduced the SPLICS $_{\mathrm{L}}$ foci (Fig. $5 \mathrm{c}, \mathrm{d}$ ). Treatment with CCCP reduced the number of fluorescent foci measured using both the SPLICS probes, suggesting that activation of PINK1/ Parkin-mediated mitophagy loosens all types of ER-mitochondria contacts.

\section{SPLICS visualizes ER-mitochondria interactions in living zebrafish neurons}

We finally wished to test if SPLICS can measure ER-mitochondria tethering in an in vivo setting. Imaging of subcellular structures in living animals, and even more in neuronal axons, is limited by the thickness and anatomical accessibility of tissues. In vivo detection of organelle contact sites is still a major challenge because of their dynamic nature and the lack of appropriate tools. To verify if SPLICS could overcome these hurdles, we expressed the new probes in $D$. rerio, specifically in Rohon-Beard (RB) sensory neurons. The correct targeting of the OMM$\mathrm{GFP}_{1-10}$ and the $\mathrm{ER}_{\mathrm{S}}-\beta_{11}$ constructs was first verified after mosaic expression in $D$. rerio embryos. The OMM-GFP ${ }_{1-10}$ signal reconstituted by complementation with a $\beta_{11}$-tagged cytosolic protein (DJ-1- $\beta_{11}$ ) fully overlapped with a mitochondrial targeted RFP (pTagRFP-mito). Analogously, injection of $\mathrm{ER}_{\mathrm{S}}-\beta_{11}$ and a cytosolic $\mathrm{GFP}_{1-10}$ resulted in fluorescence emission that co-localized with an ER marker (pDsRed2-ER) (Supplementary Figure S10), thus demonstrating that the SPLICS fragments are properly expressed, targeted and self-assembled in living zebrafish embryos. To allow tissue specific as well as equimolar expression of SPLICS, we generated an expression vector where OMM$\mathrm{GFP}_{1-10}$ and $\mathrm{ER}_{\mathrm{S}} \beta_{11}$ are linked by a P2A peptide (SPLICS $\left._{\mathrm{S}}-\mathrm{P} 2 \mathrm{~A}\right)$, an approach suitable also in zebrafish [48]. SPLICS $_{\mathrm{S}}$-P2A was placed under the control of a bidirectional UAS promoter together with a cytosolic DsRed

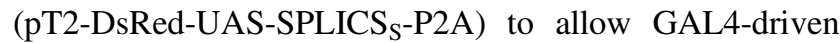
expression of the UAS promoter (Fig. 6a). The pT2-DsRed- 

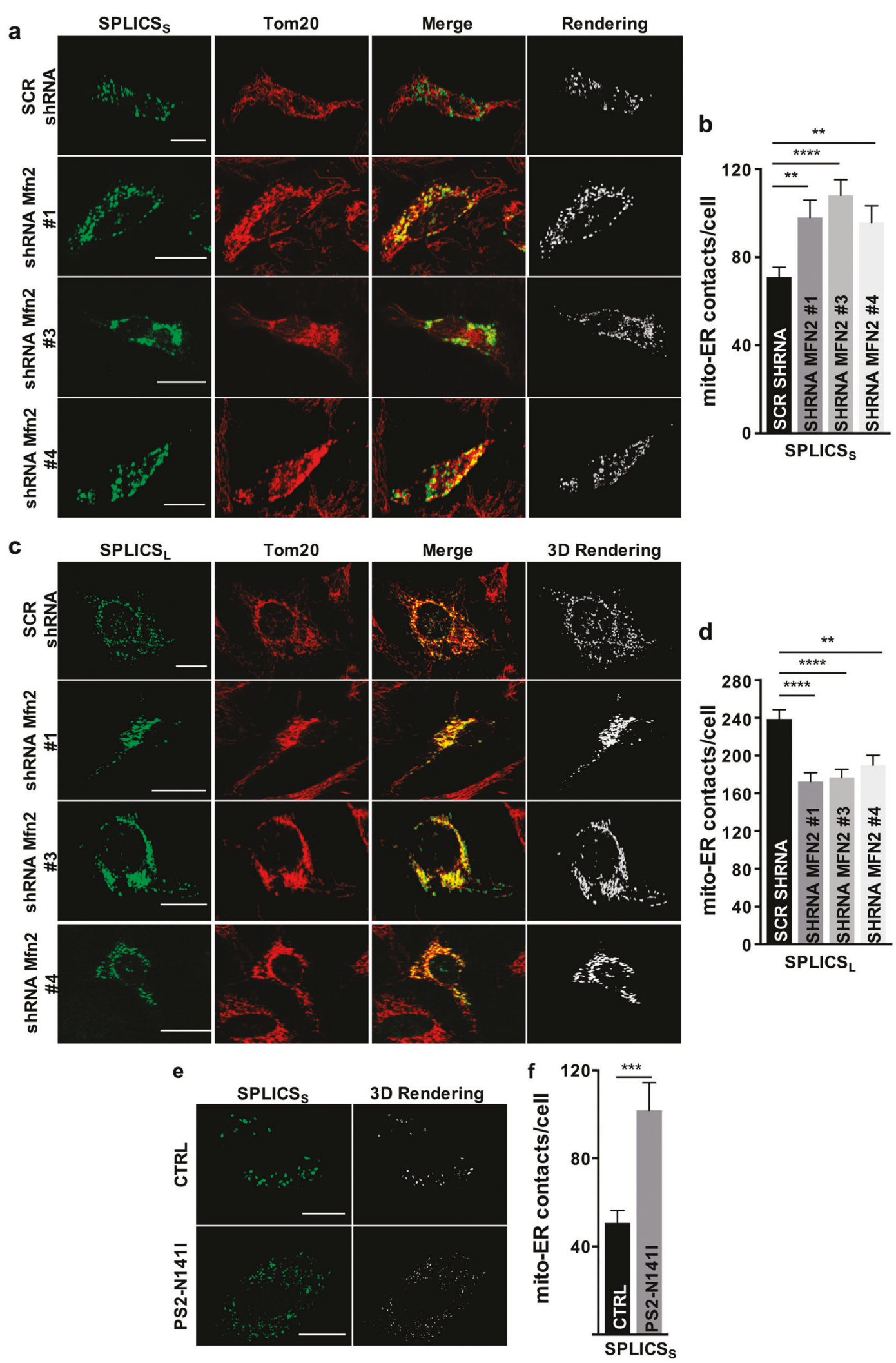

UAS-SPLICS $\mathrm{S}_{\mathrm{S}}-\mathrm{P} 2 \mathrm{~A}$ vector was then microinjected in the zebrafish s1102t:GAL4 transgenic line where GAL4 expression is restricted to RB neurons (Fig. 6b), yielding simultaneous, tissue specific expression of DsRed and SPLICS $_{S}$ (Fig. 6c). By imaging the DsRed-positive neurons, we noticed the occurrence of short-range ER 
Fig. 4 Effect of Mfn2 knockdown and mutant PS2 on ER-mitochondria interface. Immunofluorescence against mitochondria (Tom20, red) is shown in the panels on the middle. The green channel is the merge of several planes. Scale bar $20 \mu \mathrm{m}$. a Representative confocal pictures of HeLa cells expressing the SPLICS $_{S}$ probe. b Quantification of SPLICS contacts by 3D rendering of complete zstacks. Mean \pm SEM: SCR shRNA $70 \pm 4, n=76$ cells; shRNA Mfn2 \#1 $98 \pm 7, n=26$ cells; shRNA Mfn2 \#3 $108 \pm 7, n=28$ cells; shRNA Mfn2 \#4: $95 \pm 7, n=23$ cells. c Representative confocal pictures of HeLa cells expressing the SPLICS $\mathrm{L}_{\mathrm{L}}$ probe. d Quantification of SPLICS $\mathrm{L}_{\mathrm{L}}$ contacts by $3 \mathrm{D}$ rendering of complete z-stacks. Mean \pm SEM: SCR shRNA $238 \pm 10, n=30$ cells; shRNA Mfn2 $\# 1172 \pm 9$, $n=27$ cells; shRNA Mfn2 \#3 $176 \pm 8, n=29$ cells; shRNA Mfn2 \#4 $190 \pm 10, n=27$ cells. e Representative confocal pictures of human fibroblasts from a patient with the N141I mutation in PS2 (bottom panel) and an age-matched control (upper panel) expressing the SPLICS $_{S}$ probe. The green channel is the merge of several planes. Scale bar $20 \mu \mathrm{m}$. f Quantification of ER-mitochondria short contacts by $3 \mathrm{D}$ rendering of complete z-stacks. Mean \pm SEM: CTRL $50 \pm 5$, $n=20$ cells; PS2-N14I: $101 \pm 12, n=21$ cells. Data shown are the result of $2-5$ independent experiments. $* * p \leq 0.01$, *** $p \leq 0.001$, $* * * * p \leq 0.0001$

mitochondria contacts in both cell body and axons

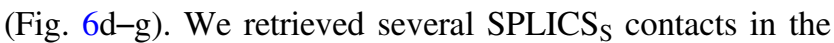
soma of RB neurons; their frequency was comparable to that observed in cultured cells. ER-mitochondria contact sites were also retrieved in $\mathrm{RB}$ axons and enriched at axonal varicosities and branching points, possibly representing axon zones with specialized functions where ER-mitochondria crosstalk is important to propagate and regulate $\mathrm{Ca}^{2+}$ signals [49-51] (arrowheads in Fig. 6f). The number of short ER-mitochondria interactions was comparable in soma and axons (Fig. 6g), suggesting that these juxtapositions are regulated by similar mechanisms in the two portions of the neuron.

\section{Discussion}

Here we report a one-step, split-GFP-based method to assess narrow and wide ER-mitochondria contact sites and their modulation and demonstrate its suitability to monitor by confocal microscopy inter-organelle interactions in human cells and in vivo in zebrafish RB neurons.

The SPLICS is versatile and sensitive and reveals short and long-range ER-mitochondria interactions and their changes upon pharmacological and genetic manipulations.

An advantage of SPLICS over the other probes relies not only on its unnerving modularity, but also on the high brightness, stability and a high threshold over background. The first feature was exploited here to generate two versions of SPLICS, one engineered to detect narrow $(\approx 8-10 \mathrm{~nm})$ and a second to image wide $(\approx 40-50 \mathrm{~nm})$ ER-mitochondria interactions [5, 13]. Although the low dissociation rate of the GFP fragments [23] could, upon reconstitution, make their association poorly reversible (implying that any reduction observed with SPLICS $_{\mathrm{S} / \mathrm{L}}$ may not reflect a dynamic decrease during time). Even if the observation of transient interactions between the ER and motile mitochondria could be limited by the time required to achieve full reconstitution of the $\mathrm{SPLICS}_{\mathrm{S} / \mathrm{L}}$, we were able to provide important insights in the biology of this interface.

SPLICS can be also adapted to monitor other types of heterotypic organelle contact sites, e.g., ER and plasma membrane (PM), mitochondria and PM, or mitochondria and endosomes/lysosomes, creating a palette of SPLICS to image inter-organelle interactions.

The physiological significance of long-range ERmitochondria contacts has not been completely defined; nevertheless, the comparison of the SPLICS $_{\mathrm{S} / \mathrm{L}}$ signals under different pathophysiological conditions indicates that ER-mitochondria tethering is heterogeneous and tightly modulated. Both ER stress and starvation increased SPLICS $_{S}$ foci while SPLICS $_{L}$ dots were decreased in number under ER stress, suggesting a spatial and functional specialization of different ER-mitochondria contact sites [27]. Changes in mitochondrial shape also affected the ER-mitochondria interface differently: Drp1 overexpression reduced SPLICS$_{\mathrm{L}}$ interactions, whereas forced mitochondrial elongation increased both SPLICS $_{\mathrm{S} / \mathrm{L}}$ foci number. Fragmentation concomitantly ensures basal $\mathrm{Ca}^{2+}$-dependent homeostatic mitochondrial functions and protects from stress responses involving $\mathrm{ER}-$ mitochondria $\mathrm{Ca}^{2+}$ crosstalk [30]. Indeed, potentiation of the ER-mitochondria interface under conditions of Drp1-dependent fragmentation can lead to mitochondrial $\mathrm{Ca}^{2+}$ overload and cell death. The reduction in SPLICS $_{\mathrm{L}}$ number is probably due to the reduction of the interface available for additional contacts and it could be relevant in the $\mathrm{Ca}^{2+}$-dependent stress responses. Of note, Drp1-dependent mitochondrial fission facilitates mitophagy whereas mitochondrial elongation inhibits mitochondrial autophagy [52]. Thus, it is tempting to speculate that mitochondria are protected from mitophagy when mitochondrial fusion is forced due to increased ER-mitochondria contacts; conversely, mitochondrial fission is accompanied by a reduction in SPLICS $_{\mathrm{L}}$ signal to favor the engulfment of mitochondria by the autophagosome.

The SPLICS described here can also help to understand the role of $\mathrm{Mfn} 2$ in ER-mitochondria juxtaposition: we observed that Mfn2 silencing led to an increase in SPLICS $_{S}$ and a decrease in SPLICS$_{\mathrm{L}}$ dots. Similar findings were observed upon the overexpression of the well-established tethering machinery VAPB/PTPIP51. Future work might therefore capitalize on spectral variant of the SPLICS $_{\mathrm{S} / \mathrm{L}}$ probes to verify if the wide interactions comprise the narrow ones, or if they occur on different areas of the organelles.

CCCP treatment in HeLa cells expressing Parkin caused a marked reduction in both SPLICS $_{S}$ and SPLICS $_{L}$ signal, 

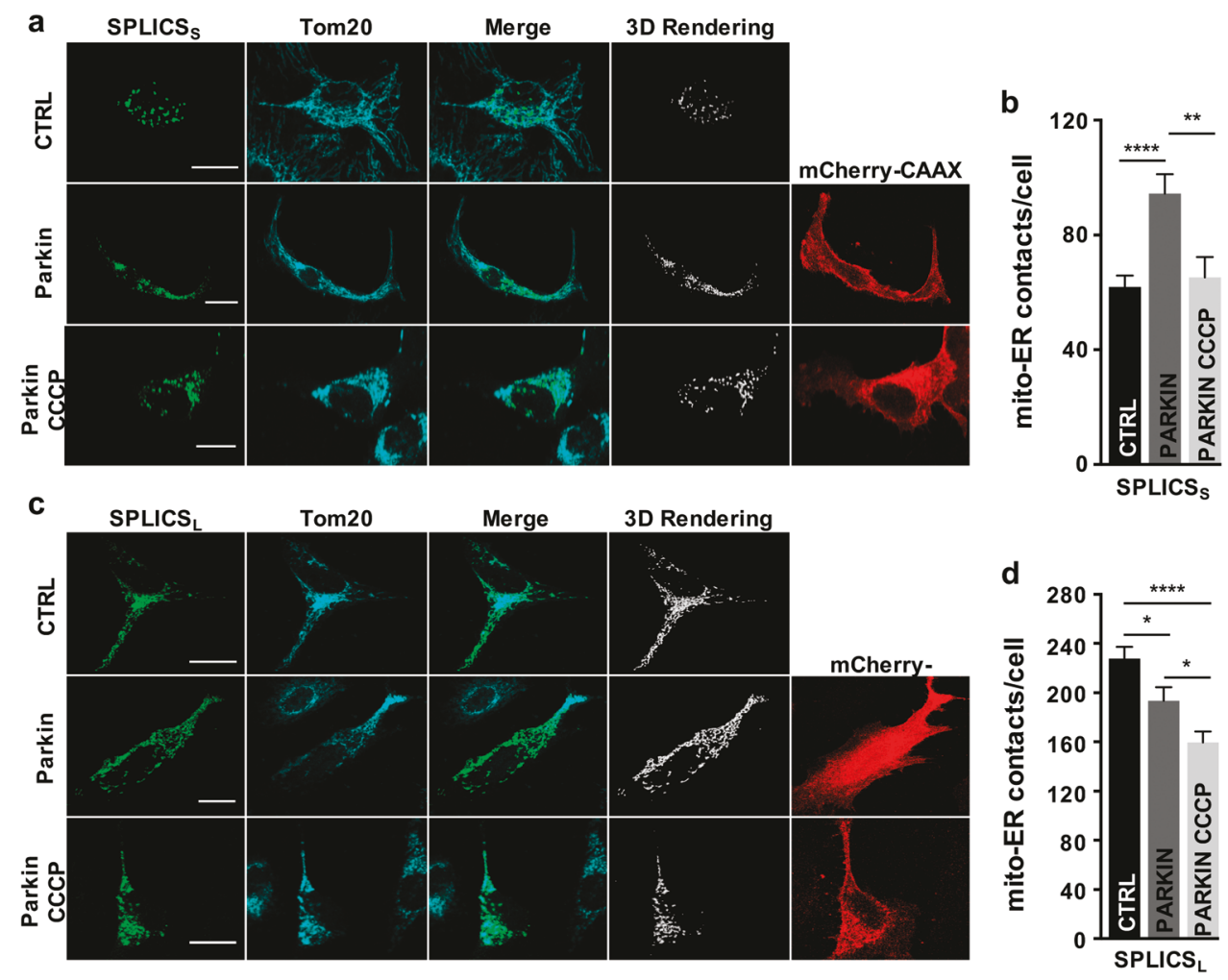

Fig. 5 Effects of Parkin on ER-mitochondria contacts. Immunofluorescence against mitochondria (Tom20, cyan) is shown in the panels on the middle. The green channel is the merge of several planes. Scale bar $20 \mu \mathrm{m}$. a Representative confocal pictures of HeLa cells expressing the SPLICS $_{S}$ probe along with Parkin-2A-mCherry-CAAX (bottom panels). b Quantification of SPLICS contacts by $3 \mathrm{D}$ rendering of complete zstacks. Mean \pm SEM: Ctrl $61 \pm 3, n=57$ cells; Parkin $94 \pm 6, n=33$

supporting a model whereby mitochondria and ER separate during mitophagy. Under basal conditions, overexpression of Parkin conversely increases ER-mitochondria tethering in several cellular models [42, 43], suggesting a housekeeping function at this interface, whereas massive Parkin activation and mitophagy induction mirrors a condition of degradation/deactivation of a handful of molecules involved in ER-mitochondria juxtaposition (e.g., VDAC, Mfn2, TOM20) [53] with consequent ER-mitochondria separation.

Despite the efforts in the analysis of the ER-mitochondria contact sites, their properties in neurons and, most importantly, in living vertebrates are still poorly characterized due to the lack of appropriate methods suitable for in vivo studies. We optimized the SPLICS for in vivo expression by generating the pT2-DsRed-UAS-SPLICS $\mathrm{S}_{\mathrm{S}}-2 \mathrm{~A}$ construct. SPLICS could report ER-mitochondria contacts in cell body and axons of RB neurons in living zebrafish embryos. ER-mitochondria contacts in the axons often localized at sites of branching, a process sustained by mitochondrial ATP [54] and $\mathrm{Ca}^{2+}$ release from internal stores [55], thus suggesting that ER-mitochondria interplay may have a role cells; Parkin CCCP $65 \pm 7, n=26$ cells. c Representative confocal pictures of HeLa cells expressing the SPLICS ${ }_{\mathrm{L}}$ probe along with Parkin-2A-mCherry-CAAX (bottom panels). d Quantification of the SPLICS $_{\mathrm{L}}$ contacts by $3 \mathrm{D}$ rendering of complete z-stacks. Mean \pm SEM: CTRL $227 \pm 9, n=44$ cells; Parkin $193 \pm 11, n=32$ cells; Parkin CCCP $159 \pm 8, n=22$ cells. Data shown are the result of 3-8 independent experiments. $* p \leq 0.05, * * p \leq 0.01$, $* * * * p \leq 0.0001$

in this process. SPLICS is a versatile tool to visualize ER-mitochondria contacts in vivo both in physiological and pathological conditions and it will be useful to explore how disease-related genes affect neuronal function and survival through the modulation of the ER-mitochondria interface.

\section{Materials and methods}

\section{Cell Lines}

HeLa and HEK293 cells (ATCC) were grown in a 5\% $\mathrm{CO}_{2}$ atmosphere in Dulbecco's modified Eagle's medium high glucose (DMEM; Euroclone), supplemented with $10 \%$ fetal bovine serum (Gibco), $100 \mathrm{U} / \mathrm{ml}$ penicillin and $100 \mathrm{mg} / \mathrm{ml}$ streptomycin. Where indicated, the cells were treated $48 \mathrm{~h}$ after transfection with $10 \mu \mathrm{M} \mathrm{CCCP}$ (SigmaAldrich), Hbss (Thermo Fisher) or $10 \mu \mathrm{g} / \mathrm{ml}$ Tunicamycin (Sigma-Aldrich) for $4 \mathrm{~h}$ at $37{ }^{\circ} \mathrm{C}$, in a $5 \% \mathrm{CO}_{2}$ atmosphere. Mock cells were maintained in growth medium, which was changed simultaneously with the beginning of treatments. Female FAD-PS2-N141I fibroblasts (Coriell 
Fig. 6 Expression of the SPLICS $_{S}$ probe in living zebrafish embryos. a Schematic depiction of the bidirectional construct that allows detection of DsRed and SPLICS S $^{-P 2 A}$ in a Gal4-dependent manner. The $2 \mathrm{~A}$ peptide guarantees the generation of an equimolar amount of the two spGFP fragments. b Experimental setting used to image ER-mitochondria contacts in zebrafish embryos: a schematic drawing of RB neurons is shown on the right. c Representative image of a $1 \mathrm{dpf}$ s1102t:GAL4 embryo injected with the pT2DsRed-UAS-SPLICS $_{\mathrm{S}}$-P2A construct. d Live imaging of short ER-mitochondria contacts in RB neurons of s1102t:GAL4 zebrafish embryos. The picture is the merge of several planes. The 3D rendering of the $\mathrm{z}$-stack is shown on the right. Scale bar: $15 \mu \mathrm{m}$. e Quantification of SPLICS $_{S}$ contacts in the cell body of RB neurons by 3D rendering of complete z-stacks. Mean \pm SEM: $22 \pm 1, n=28$ cells from 11 fish. f Live imaging of SPLICS $S_{S}$ contacts in the axons of RB neurons. The picture is the merge of several planes. The 3D rendering of the complete z-stack is shown on the right. Scale bar: $15 \mu \mathrm{m}$. g Quantification of the density of SPLICS $_{S}$ contacts in the cell body and the axons of RB neurons. Mean \pm SEM: RB soma: $0.17 \pm 0.01, n=28$ cells from 11 fish; RB axon: $0.14 \pm$ $0.01, n=20$ cells from 6 fish. Data shown are the result of two independent experiments
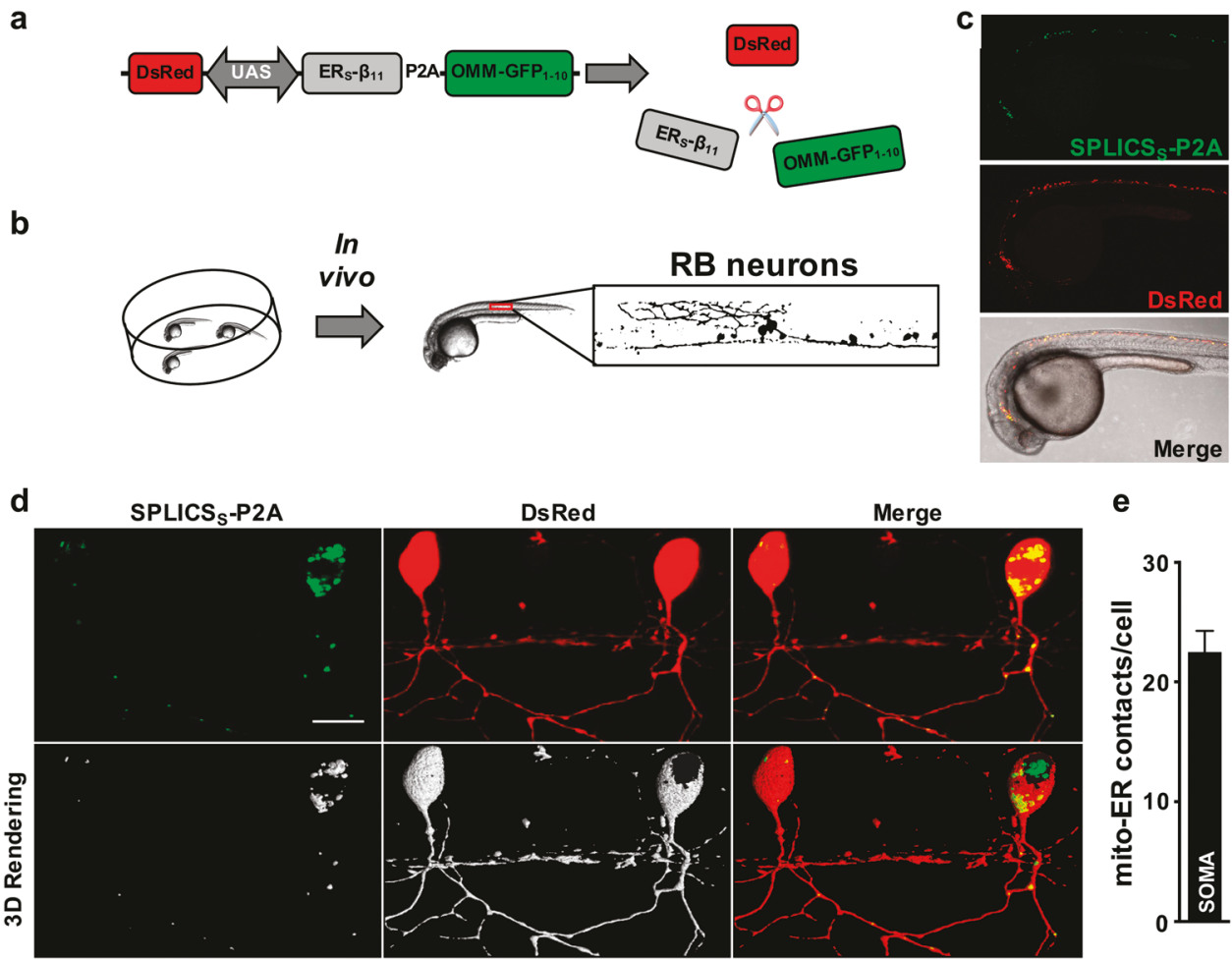

e

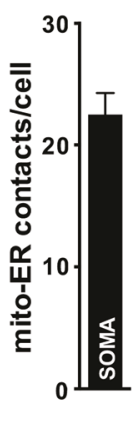

f

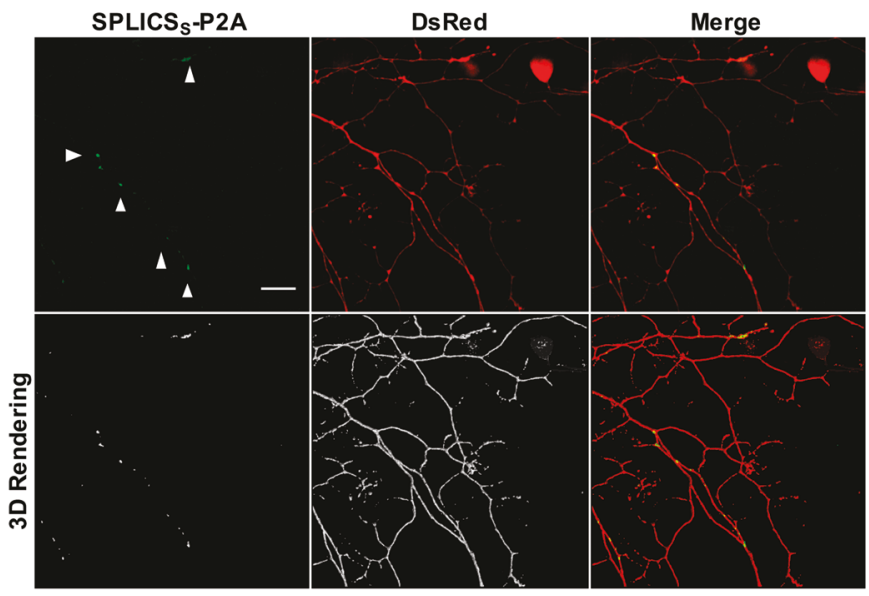

g

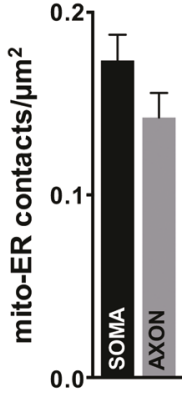

Institute, AG09908) and age/sex-matched control fibroblasts (Coriell Institute, AG08269) were grown in DMEM containing FCS $(15 \%)$, L-glutamine $(2 \mathrm{mM})$, penicillin $(100 \mathrm{U} / \mathrm{ml}$, Euroclone), and streptomycin $(100 \mu \mathrm{g} / \mathrm{ml}$, Euroclone).

\section{Zebrafish husbandry and transgenic lines}

All animal experiments were conducted as previously reported [56]. Adult fish were maintained and raised in 51 tanks with freshwater at $28^{\circ} \mathrm{C}$ with a $12 \mathrm{~h} \mathrm{light} / 12 \mathrm{~h}$ dark cycle. Zebrafish embryos were obtained from spontaneous spawnings. To obtain fish selectively expressing Gal4 in
Rohon-Beard neurons (s1102t:GAL4), Et(E1b:GAL4VP16)s1102t; $\operatorname{Tg}$ (UAS-E1b:Kaede)s1999t fish (ZIRC, ZL1384) were outcrossed with wild-type (wt) fish [57] and the fluorescent offspring was discarded. The remaining fish were genotyped from fin clips with primers specific for GAL4. To perform experiments, both wt and s1102t:GAL4 fish were used. All experiments were conducted on $24 \mathrm{~h}$ post fertilization (hpf) embryos.

\section{Transfection}

Twelve hours before transfection, HeLa cells were seeded onto $13 \mathrm{~mm}$ glass coverslips and allowed to grow to $50 \%$ 
confluence. Cells were transfected by calcium phosphate [58]. For co-transfection, the two SPLICS ER and mitochondrial fragments were in a 1.5:2 ratio with the overexpressed protein of interest. Human fibroblasts were electroporated by using a NeonTM transfection system (Life Technologies), according to the manufacturer's instruction.

\section{Cloning and fusion plasmid construction}

Humanized $\mathrm{GFP}_{1-10}\left(\mathrm{GFP}_{1-10(\mathrm{~h})}\right)$ was PCR amplified from the GPI-GFP ${ }_{1-10(\mathrm{~h})}$ kindly provided by Prof. Fabien Pinaud, Department of Biological Sciences and Chemistry, University of Southern California [59], to insert the Tom20 N33 targeting sequence [60] by using the OMM-GFP ${ }_{1-10}$ For. (TCGAATTCATGGTGGGCCGGAACAGCGCCATCGC CGCGGGCGTGTGCGGTGCCCTCTTCATAGGGTACT GCATCTACTTTGACCGCAAAAGGCGGAGTGACCC CAACTCCAAAGGAGAAG) and Rev. (AC TTCTCACT CGAGTTATGTTCCTTTTTCATTTGGATCTTTGCTCA GG) primers and inserted into the vector pcDNA3 between the EcoRV and XhoI restriction sites. The chimeric sequences composed by the minimal Sac1 ER targeting sequence and the Sac1 ER targeting sequence containing additional $267 \mathrm{bp}$ of the helix-FRB fragment (derived from the pEGFP-C3/CFP-HA-FRB-helix-ER plasmid [5]) $\left(\mathrm{ER}_{\mathrm{L}^{-}}\right.$ $\beta_{11}$ ) were fused with the $\beta_{11}$ tag to generate the $\mathrm{ER}_{\mathrm{s}}-\beta_{11}$ and the $\mathrm{ER}_{\mathrm{L}}-\beta_{11}$ constructs, respectively. The constructs were chemically synthetized (Thermo Fisher) by using the EcoRI/NotI and the EcoRV/XhoI restriction sites of pCDNA3.1(+), respectively. The sequence encoding for $\beta_{11}$, flanked by different multi cloning sites, has been inserted into the commercial vectors pCDNA6.5/V5-DEST (Invitrogen), in order to obtain a backbone that could easily allow fusion of the $\beta_{11}$ fragment with the protein of interest. Kate was amplified with the Kate For. (AAAAAAGCTTATGGTGAGCGAGC) and the Kate Rev. (TTTTTG GTACCTCATCTGTGCC) primers using as a template mKate2-pcDNA3.1 (pEVROGEN) and inserted in pDEST$\beta_{11}$ to generate Kate $\beta_{11}$. To generate a vector simultaneously expressing (through a $2 \mathrm{~A}$ peptide) Parkin and membrane mCherry (CAAX-mCherry), the coding sequence of Parkin [42] was amplified with the primers Parkin For. (ACGCGGATCCGCCACCATGATAGTGTT TGTCAGGTT) and Rev. (TTCCCCCGGGCA CGTCGAA CCAGTGGTCC). The PCR products were purified using the GenElute Gel Extraction Kit (Sigma), digested with BamHI and SmaI and then ligated into the pSYC-187 vector (Addgene) digested with the same restriction enzymes. The constructs codifying for Drp1 WT and K38A [30, 61, 62] and pmTurquoise2-ER were kindly donated by Diego De Stefani (Department of Biomedical Sciences, University of Padova). The pCIneo-PTPIP51-HA and the pCIneo-VAPB-
Myc were kindly provided by Professor Christopher C.J. Miller (Department of Neuroscience and Department of Basic and Clinical Neurosciences, King's College London). Where indicated, mitochondria are labeled with a pTagRFPmito construct (Evrogen). To knockdown Mfn2, three different shRNAs against Mfn2 were used (SureSilencing shRNA Plasmid, Hygromycin Gene: Mfn2; Refseq Accession \#:NM_014874; Clone \#1: AGAGGCGGTTCGACT CATCAT; Clone \#3: TGATGTG GCCCAACTCTAAGT; Clone \#4: CCAGTAGTCCTCAA GGTTTAT; Scramble: GGAATCTCATTCGATGCATA C). The aminoacid sequences of the ER anchored probes are: MRDHMVLHEYVNAAGITGGDGGSGGGSKLRVF LALPIIMVVAFSMCIICLLMAGDTWTETLAYVLFWG VASIGTFFIILYNGKDFVDAPRLVQKEKID (the Short) and MRDHMVLHEYVNAAGITGGDGGSGGGSKLMW HEGLEEASRLYF GERNVKGMFEVLEPLHAMMERGP QTLKETSFNQAYGRDLMEAQEWCRKYMKSGNVKD LTQAWDLYYHVFRRISKQGSEAAAREAAARGGASG AGAGAGAILNSRVFLALPIIMVVAFSMCIICLLMAGD TWTETLAYVLFWGVASIGTFFIILYNGKDFVDAPRLV QKEKID (the Long).

\section{Zebrafish constructs}

To properly express SPLICS in zebrafish, the $\mathrm{ER}_{\mathrm{S}^{-}} \beta_{11}$ and the OMM-GFP ${ }_{1-10}$ coding sequences were respectively cloned upstream and downstream of a viral 2A peptide sequence (pSYC-181, Addgene), previously reported to be cleaved within the cell to generate an equimolar amount of the two genes [48]. The fragment encoding for the OMMGFP $_{1-10}$ was excised from pcDNA3 with EcoRI and XhoI and ligated into the pSYC-181 vector digested with the same enzymes. Since these sites cloned the OMM-GFP ${ }_{1-10}$ fragment out of frame, we mutagenized the resulting vector in order to re-establish the correct frame, using the primer OMM-GFP $_{1-10}$ mut. (CCCTGGACCTAGATCTGAATTC ATGGTGGGCC). ER $_{S}$ was amplified from pcDNA3 using the primers $\mathrm{ER}_{\mathrm{S}}-\beta_{11} 2 \mathrm{~A}$ For. (ACGCGGATCCGCCAC CATGCGGGACCACATGGTG C) and Rev. (TTCCCCCG GGGTCGATCTTCTCTTT), which introduce, respectively, a BamHI-Kozak sequence and a SmaI site at the $5^{\prime}$ and $3^{\prime}$ ends of the $\mathrm{ER}_{\mathrm{S}}$ coding sequence. The PCR product was purified, digested with both BamHI and SmaI and then ligated into the pSYC-181-OMM-GFP ${ }_{1-10}$ vector, previously digested with the same restriction enzymes. We refer to this plasmid as $\mathrm{pSYC}$-SPLICS $\mathrm{S}-\mathrm{P} 2 \mathrm{~A}$. To selectively express the SPLICS $_{\mathrm{S}}-\mathrm{P} 2 \mathrm{~A}$ in zebrafish neurons, we exploited the Gateway technology to generate the pT2DsRed-UAS-SPLICS $\mathrm{S}_{\mathrm{S}} \mathrm{P} 2 \mathrm{~A}$ vector. Briefly, the fragment encoding for the SPLICS $S_{S}-\mathrm{P} 2 \mathrm{~A}$ was excised from the pSYC-SPLICS $_{\mathrm{S}}-\mathrm{P} 2 \mathrm{~A}$ vector with BamHI and $\mathrm{Xba \textrm {I }}$ and cloned into the BamHI-XbaI sites of the pME-MCS vector 
(Tol2 kit). The resulting pME-SPLICS $\mathrm{S}_{\mathrm{S}} \mathrm{P} 2 \mathrm{~A}$ was recombined through the LR reaction with the pT2dDESTpADs Red.T4E1bUASE1bGWR1R2pA vector (kindly donated by Christian Haas, Ludwig-Maximilians University Munich). The resulting pT2-DsRed-UAS-SPLICS $\mathrm{S}_{\mathrm{S}}-\mathrm{P} 2 \mathrm{~A}$ plasmid was injected into 1-2 cells stage s1102t:GAL4 embryos. To label mitochondria and the endoplasmic reticulum, the pTagRFP-mito (Evrogen) and the pDsRed2-ER (Clontech) plasmids were, respectively, injected in WT eggs. For injections, all plasmids were diluted in Danieau solution $(58 \mathrm{mM} \mathrm{NaCl}, 0.7 \mathrm{mM} \mathrm{KCl}, 0.4 \mathrm{mM} \mathrm{MgSO}, 0.6 \mathrm{mM} \mathrm{Ca}$ $\left(\mathrm{NO}_{3}\right)_{2}, 5 \mathrm{mM}$ HEPES $\left.\mathrm{pH} 7.6\right)$ and $0.5 \%$ phenol red.

\section{Immunocytochemistry}

To image cells over-expressing a protein of interest, transfected cells plated on $13 \mathrm{~mm}$ glass coverslips were fixed 48-72 $\mathrm{h}$ post transfection with $3.7 \%$ formaldehyde in phosphate-buffered saline (PBS; $140 \mathrm{mM} \mathrm{NaCl}, 2 \mathrm{mM} \mathrm{KCl}$, $1.5 \mathrm{mM} \mathrm{KH}_{2} \mathrm{PO}_{4}, 8 \mathrm{mM} \mathrm{Na} \mathrm{HPO}_{4}$, pH 7.4) for $20 \mathrm{~min}$ and washed three times with PBS. Cell permeabilization was performed by $20 \mathrm{~min}$ incubation in $0.1 \%$ Triton X-100/PBS followed by $30 \mathrm{~min}$ wash in $1 \%$ gelatin/PBS (type IV, from bovine skin, Sigma) and $15 \mathrm{~min}$ wash in PBS at room temperature (RT). The coverslips were then incubated for $90 \mathrm{~min}$ at $37^{\circ} \mathrm{C}$ with the specific primary antibody diluted 1:20 in PBS (Tom20: Santa Cruz Biotech., Cat\#sc-11415; Parkin: Santa Cruz Biotech., Cat\#sc-32282; Drp1: BD Biosciences, Cat\#611113; mtHSP60: Abcam, Cat\#ab82520; Calreticulin: Thermo Fisher, Cat\#PA3-900; Myc: Millipore, Cat\#05-724; HA: Cell Signalling, Cat\#3724S). Further washing steps with gelatine and PBS were repeated as mentioned before to remove the excess of primary antibody. Staining was revealed by the incubation with specific AlexaFluor secondary antibodies (Thermo Fisher: Goat anti-Rabbit IgG AlexaFluor 405, Cat\#A-31556; Goat anti-Rabbit IgG AlexaFluor 594, Cat\#A11012; Donkey anti-Goat IgG AlexaFluor 633, Cat\#A-21082; Goat anti-Mouse IgG AlexaFluor 633, Cat\#A-21050; Goat anti-Mouse IgG AlexaFluor 488, Cat\#A-11001; Goat antiRabbit IgG AlexaFluor 488, Cat\#A-11008) for $45 \mathrm{~min}$ at RT (1:50 dilution in PBS; 1:20 only for Goat anti-Rabbit IgG AlexaFluor 405). After further washing steps, coverslips were mounted using Mowiol 4-88 (Sigma). The coverslips were observed at the SP5 Leica confocal microscope at lasers wavelength of $405,458,488,543,555$, and $633 \mathrm{~nm}$.

\section{Electron microscopy}

Cells were immunogold labeled by the Tokuyasu technique [63]. Briefly, cells were fixed in $2 \%$ paraformaldehyde, $0.2 \%$ glutaraldehyde in $0.1 \mathrm{M}$ phosphate buffer (PB) for $1 \mathrm{~h}$ at RT. Next cells were gently scraped in $1 \%$ gelatin and embedded in $12 \%$ gelatin in PB. Gelatin squared blocks were infiltrated overnight in $2.3 \mathrm{M}$ sucrose, mounted on aluminum pins, and frozen in liquid nitrogen. Ultrathin cryosections of $70 \mathrm{~nm}$ were cut with a Leica FC7 ultramicrotome (Leica Microsystems, Germany), collected on formvar, carbon-coated 150 mesh copper grids and immunolabeled with anti-GFP (Abcam, Cat\#ab290) and $10 \mathrm{~nm}$ Protein A-gold (Utrecht University, The Netherlands). Grids were then contrasted for 10 min on ice in a solution of $1.8 \%$ methylcellusose $/ 0.4 \%$ uranyl acetate, air dried on wire loops and observed in a ZEISS Leo912AB (Zeiss, Oberkochen, Germany). Images were acquired using a $2 \mathrm{Kx} 2 \mathrm{~K}$ bottom mounted slow-scan Proscan camera (Scheuring, Germany) controlled by the EsivisionPro 3.2 (Soft Imaging System, Münster, Germany).

\section{Image acquisition and processing}

Cells were generally imaged $48-72 \mathrm{~h}$ after transfection with a Leica TSC SP5 inverted confocal microscope, using either a HCX PL APO 63X/numerical aperture $1.40-0.60$ or a HCX PL APO $\times 100 /$ numerical aperture 1.4 oil-immersion objective. Images were acquired by using the Leica AS software. To count ER-mitochondria contacts, a complete Z-stack of the cell was acquired every $0.29 \mu \mathrm{m}$. Z-stacks were processed using Fiji [64]: images were first convolved, and then filtered using the Gaussian Blur filter. A 3D reconstruction of the resulting image was obtained using the Volume J plugin (http://bij.isi.uu.nl/vr.htm). A selected face of the 3D rendering was then thresholded and used to count ER-mitochondria contact sites.

\section{Zebrafish imaging}

At $24 \mathrm{hpf}$, embryos were screened for fluorescence, dechorionated and fixed or anesthetised according to the experiment. To image the co-localization of $E_{S}-\beta_{11}$ and OMM-GFP $_{1-10}$ with mitochondria and the ER, fish were fixed for $2 \mathrm{~h}$ at RT with 4\% PFA, then washed with PBS and mounted in low melting agarose (1.3\%, Euroclone) on glass coverslips.

For in vivo imaging, fish were anesthetised and mounted on $35 \times 10 \mathrm{~mm}$ glass bottom Petri dishes (Ted Pella, INC. Prod. No. 14023-20) in low melting agarose (1.3\%, EuroClone). Fish water containing tricaine methanesulfonate $0.61 \mathrm{mM}$ (Sigma) was added in the Petri dishes, in order to keep fish anesthetised. Mounted fish were imaged at RT $\left(20-23^{\circ} \mathrm{C}\right)$ using a Leica TSC SP5 inverted confocal microscope, using either a HCX PL APO $\times 63 /$ numerical aperture $1.40-0.60$ or a HCX PL APO $\times 100$ /numerical aperture 1.4 oil-immersion objective. To count ER-mito contacts, a complete $\mathrm{z}$-stack of the cell was acquired every $0.29 \mu \mathrm{m}$. To acquire a representative image of a whole fish expressing pT2-DsRed-UAS-SPLICS S $_{-}$-PA, a $\times 10 \mathrm{HCPX}$ PL Fluotar NA 0.3 objective was used. 


\section{$\mathrm{Ca}^{2+}$ measurements}

$\mathrm{Ca}^{2+}$ measurements were performed by co-transfecting HeLa cells in a six-well plate with low-affinity mitochondrial aequorin (mtAeqmut) with the two SPLICS moieties in a 1.5:1.5:1 ratio favouring SPLICS. Forty-eight hours post transfection, cells were re-plated into a 96-wells plate (PerkinElmer). mtAeqmut was reconstituted by incubating cells for $1.5 \mathrm{~h}$ with $5 \mu \mathrm{M}$ coelenterazine (Santa Cruz Biotech.) in modified Krebs Ringer Buffer (KRB: $125 \mathrm{mM}$ $\mathrm{NaCl}, 5 \mathrm{mM} \mathrm{KCl}, 400 \mathrm{mM} \mathrm{KH}{ }_{2} \mathrm{PO}_{4}, 1 \mathrm{mM} \mathrm{MgSO}{ }_{4}, 20$ $\mathrm{mM}$ Hepes, $\mathrm{pH}$ 7.4) supplemented with $5 \mathrm{mM}$ glucose at $37^{\circ} \mathrm{C}$. Luminescence measurements were carried out using a PerkinElmer EnVision plate reader equipped with two injector units. After reconstitution, cells were placed in 70 $\mu \mathrm{l}$ of KRB solution and luminescence from each well was measured for $1 \mathrm{~min}$. During the experiment, $100 \mu \mathrm{M}$ histamine at the final concentration were first injected to activate $\mathrm{Ca}^{2+}$ transients, and then a hypotonic, $\mathrm{Ca}^{2+}$-rich, digitonin-containing solution was added to discharge the remaining aequorin pool. Output data were analyzed and calibrated with a custom-made macro-enabled Excel workbook.

\section{Western blot}

HeLa cells were seeded in a six-well plate and transfected with three different shRNAs against Mfn2. A scramble shRNA was used as control. At $48 \mathrm{~h}$ post transfection, cells were washed with PBS and proteins were extracted for $20 \mathrm{~min}$ using ice cold lysis buffer $(50 \mathrm{mM}$ Tris- $\mathrm{HCl} \mathrm{pH}$ 7.4, $150 \mathrm{mM} \mathrm{NaCl}, 10 \mathrm{mM}$ EGTA, $1 \%$ Triton X-100, 1 $\mathrm{mM}$ protease inhibitor cocktail (Sigma)). Samples were then centrifuged at $10,000 \mathrm{rpm}$ for $10 \mathrm{~min}$ at $4{ }^{\circ} \mathrm{C}$. Protein concentration was determined by the Bradford assay (BioRad). Samples were separated on a $4-15 \%$ MiniPROTEAN TGX ${ }^{\text {TM }}$ Precast Protein Gels (Bio-Rad) and blotted using a Immobilon-PSQ PVDF Membrane (Merck Millipore). The membrane was blocked for $1 \mathrm{~h}$ at RT using $5 \%$ non-fat dried milk in TBST $(20 \mathrm{mM}$ Tris- $\mathrm{HCl}, \mathrm{pH} 7.4$, $150 \mathrm{mM} \mathrm{NaCl}, 0.05 \%$ Tween-20) and incubated with primary antibodies (Mfn2: Abcam, Cat\#ab50838, 1:1000 in TBST, overnight at $4{ }^{\circ} \mathrm{C} ; \beta$-actin: Sigma, Cat\#A5441, 1:30,000 in TBST, $2 \mathrm{~h}$ at RT; Myc: Millipore, Cat\#05-724, 1:1000 in TBST, overnight at $4{ }^{\circ} \mathrm{C}$; HA: Cell Signalling, Cat\#3724 S, 1:1000 in TBST, overnight at $4{ }^{\circ} \mathrm{C}$ ). After three washing steps in TBST, detection was obtained by incubating the membrane with secondary horseradish peroxidase-conjugated antibodies (Santa Cruz Biotech.: Goat anti-Rabbit IgG-HRP, Cat\#sc-2004; Goat anti-Mouse IgG-HRP, Cat\#sc-2005; 1:2000 in TBST) for $1 \mathrm{~h}$ at RT and by incubation with the Luminata HRP substrate (Merck Millipore).

\section{Statistical analysis}

Results shown are mean values \pm SEM. Student's unpaired two-tailed $t$-test was used for comparisons involving two groups when sample followed a Gaussian distribution, otherwise Mann-Whitney test was used. Differences between groups were considered significant when $p \leq 0.05$. All statistical analyses were performed using GraphPad Prism version 6.00 for Mac OS X, GraphPad Software (La Jolla, California, USA). The exact values of $n$ and their means are indicated in the figure legends. $* p \leq 0.05$, $* * p \leq$ $0.01, * * * p \leq 0.001, * * * * p \leq 0.0001$.

Acknowledgements We are deeply indebted with Prof. F. Pinaud (University of Southern California) for kindly providing the humanized GFP $_{1-10}$ construct. We thank Prof. G. Hajnoczky (Thomas Jefferson University, Philadelphia) for kindly providing the plasmid pEGFP-C3/ CFP-HA-FRB-helix-ER. We thank Prof. C. C.J. Miller (King's College London) for kindly providing the PTPIP51-HA and the VAPB-Myc expression vectors. We thank Prof. R. Rizzuto (University of Padova), Dr. D. De Stefani (University of Padova) and Prof. G. Szabadkai (University College London) for helpful discussions, we also thank Prof. F. Argenton and the Zebrafish Facility of the Department of Biology, University of Padova and Dr. A. Raimondi (Advanced Light and Electron Microscopy BioImaging Center ALEMBIC, San Raffaele Scientific Institute). This research was supported by grants from the Ministry of University and Research (Bando SIR 2014 no. RBSI14C65Z to T.C.; FIRB RBAP11Z3YA_005 to L.S.), from the University of Padova (Progetto Giovani 2012 no. GRIC128SP0 to T.C., Progetto di Ateneo 2016 no. CALI_SID16_01 to T.C., Progetto di Ateneo 2015 no. CPDA153402 to M.B.), from Cariparo Starting Grant 2016 AIFbiol to M.G., from the EU Joint Programme in Neurodegenerative Disease (2015-2018, "Cellular Bioenergetics in Neurodegenerative Diseases: A System-Based Pathway and Target Analysis") to P.P., from the European Union (ERC FP7-282280, FP7 CIG PCIG13GA-2013-618697 to L.S.) and from Telethon (GGP02016 to L.S.).

\section{Compliance with ethical standards}

Conflict of interest The authors declare that they have no conflict of interest.

Open Access This article is licensed under a Creative Commons Attribution 4.0 International License, which permits use, sharing, adaptation, distribution and reproduction in any medium or format, as long as you give appropriate credit to the original author(s) and the source, provide a link to the Creative Commons license, and indicate if changes were made. The images or other third party material in this article are included in the article's Creative Commons license, unless indicated otherwise in a credit line to the material. If material is not included in the article's Creative Commons license and your intended use is not permitted by statutory regulation or exceeds the permitted use, you will need to obtain permission directly from the copyright holder. To view a copy of this license, visit http://creativecommons. org/licenses/by/4.0/.

\section{References}

1. Prinz WA. Bridging the gap: membrane contact sites in signaling, metabolism, and organelle dynamics. J Cell Biol. 2014;205:759-69. 
2. Eisenberg-Bord M, Shai N, Schuldiner M, Bohnert M. A tether is a tether is a tether: tethering at membrane contact sites. Dev Cell.2016;39:395-409.

3. Murley A, Nunnari J. The emerging network of mitochondriaorganelle contacts. Mol Cell.2016;61:648-53.

4. Giacomello M, Pellegrini L. The coming of age of the mitochondria-ER contact: a matter of thickness. Cell Death Differ. 2016;23:1417-27.

5. Csordas G, Renken C, Varnai P, Walter L, Weaver D, Buttle KF, et al. Structural and functional features and significance of the physical linkage between ER and mitochondria. J Cell Biol. 2006;174:915-21.

6. Tubbs E, Rieusset J. Study of endoplasmic reticulum and mitochondria interactions by in situ proximity ligation assay in fixed cells. J Vis Exp. 2016;118. doi: 10.3791/54899. PubMed PMID: 28060261.

7. Gomez-Suaga P, Paillusson S, Stoica R, Noble W, Hanger DP, Miller CC. The ER-mitochondria tethering complex VAPBPTPIP51 regulates autophagy. Curr Biol. 2017;27:371-85.

8. Stoica R, Paillusson S, Gomez-Suaga P, Mitchell JC, Lau DH, Gray EH, et al. ALS/FTD-associated FUS activates GSK-3beta to disrupt the VAPB-PTPIP51 interaction and ER-mitochondria associations. EMBO Rep. 2016;17:1326-42.

9. Paillusson S, Gomez-Suaga P, Stoica R, Little D, Gissen P, Devine MJ, et al. alpha-Synuclein binds to the ER-mitochondria tethering protein VAPB to disrupt $\mathrm{Ca} 2+$ homeostasis and mitochondrial ATP production. Acta Neuropathol. 2017; 134:129-49.

10. Rizzuto R, Pinton P, Carrington W, Fay FS, Fogarty KE, Lifshitz $\mathrm{LM}$, et al. Close contacts with the endoplasmic reticulum as determinants of mitochondrial $\mathrm{Ca} 2+$ responses. Science. 1998; 280:1763-6.

11. de Brito OM, Scorrano L. Mitofusin 2 tethers endoplasmic reticulum to mitochondria. Nature. 2008;456:605-10.

12. Naon D, Zaninello M, Giacomello M, Varanita T, Grespi F, Lakshminaranayan $\mathrm{S}$, et al. Critical reappraisal confirms that Mitofusin 2 is an endoplasmic reticulum-mitochondria tether. Proc Natl Acad Sci USA. 2016;113:11249-54.

13. Filadi R, Greotti E, Turacchio G, Luini A, Pozzan T, Pizzo P. Mitofusin 2 ablation increases endoplasmic reticulummitochondria coupling. Proc Natl Acad Sci USA. 2015;112: E2174-81.

14. Alford SC, Ding Y, Simmen T, Campbell RE. Dimerizationdependent green and yellow fluorescent proteins. ACS Synth Biol. 2012;1:569-75.

15. Toulmay A, Prinz WA, A conserved membrane-binding domain targets proteins to organelle contact sites. J Cell Sci.2012;125(Pt 1):49-58.

16. MacDonald ML, Lamerdin J, Owens S, Keon BH, Bilter GK, Shang $\mathrm{Z}$, et al. Identifying off-target effects and hidden phenotypes of drugs in human cells. Nat Chem Biol. 2006;2:329-37.

17. Csordas G, Varnai P, Golenar T, Roy S, Purkins G, Schneider TG, et al. Imaging interorganelle contacts and local calcium dynamics at the ER-mitochondrial interface. Mol Cell. 2010;39:121-32.

18. Ravikumar B, Duden R, Rubinsztein DC. Aggregate-prone proteins with polyglutamine and polyalanine expansions are degraded by autophagy. Hum Mol Genet. 2002;11:1107-17.

19. Ravikumar B, Vacher C, Berger Z, Davies JE, Luo S, Oroz LG, et al. Inhibition of mTOR induces autophagy and reduces toxicity of polyglutamine expansions in fly and mouse models of Huntington disease. Nat Genet. 2004;36:585-95.

20. Noda T, Ohsumi Y. Tor, a phosphatidylinositol kinase homologue, controls autophagy in yeast. J Biol Chem. 1998;273: 3963-6.

21. Kornmann B, Currie E, Collins SR, Schuldiner M, Nunnari J, Weissman JS, et al. An ER-mitochondria tethering complex revealed by a synthetic biology screen. Science. 2009;325: 477-81.

22. Wang PT, Garcin PO, Fu M, Masoudi M, St-Pierre P, Pante N, et al. Distinct mechanisms controlling rough and smooth endoplasmic reticulum contacts with mitochondria. J Cell Sci. 2015;128:2759-65.

23. Cabantous S, Terwilliger TC, Waldo GS. Protein tagging and detection with engineered self-assembling fragments of green fluorescent protein. Nat Biotechnol. 2005;23:102-7.

24. Pedelacq JD, Cabantous S, Tran T, Terwilliger TC, Waldo GS. Engineering and characterization of a superfolder green fluorescent protein. Nat Biotechnol. 2006;24:79-88.

25. Cabantous S, Waldo GS. In vivo and in vitro protein solubility assays using split GFP. Nat Methods. 2006;3:845-54.

26. Bravo R, Vicencio JM, Parra V, Troncoso R, Munoz JP, Bui M, et al. Increased ER-mitochondrial coupling promotes mitochondrial respiration and bioenergetics during early phases of ER stress. J Cell Sci.2011;124(Pt 13):2143-52.

27. Bravo-Sagua R, Lopez-Crisosto C, Parra V, Rodriguez-Pena M, Rothermel BA, Quest AF, et al. mTORC1 inhibitor rapamycin and ER stressor tunicamycin induce differential patterns of ERmitochondria coupling. Sci Rep. 2016;6:36394.

28. Rambold AS, Kostelecky B, Elia N, Lippincott-Schwartz J. Tubular network formation protects mitochondria from autophagosomal degradation during nutrient starvation. Proc Natl Acad Sci USA.2011;108:10190-5.

29. Gomes LC, Di Benedetto G, Scorrano L. During autophagy mitochondria elongate, are spared from degradation and sustain cell viability. Nat Cell Biol. 2011;13:589-98.

30. Szabadkai G, Simoni AM, Chami M, Wieckowski MR, Youle RJ, Rizzuto R. Drp-1-dependent division of the mitochondrial network blocks intraorganellar $\mathrm{Ca} 2+$ waves and protects against $\mathrm{Ca} 2$ + -mediated apoptosis. Mol Cell. 2004;16:59-68.

31. Hamasaki M, Furuta N, Matsuda A, Nezu A, Yamamoto A, Fujita $\mathrm{N}$, et al. Autophagosomes form at ER-mitochondria contact sites. Nature. 2013;495:389-93.

32. Sugiura A, Nagashima S, Tokuyama T, Amo T, Matsuki Y, Ishido $\mathrm{S}$, et al. MITOL regulates endoplasmic reticulum-mitochondria contacts via Mitofusin2. Mol Cell. 2013;51:20-34.

33. Chen Y, Csordas G, Jowdy C, Schneider TG, Csordas N, Wang $\mathrm{W}$, et al. Mitofusin 2-containing mitochondrial-reticular microdomains direct rapid cardiomyocyte bioenergetic responses via interorganelle $\mathrm{Ca}(2+)$ crosstalk. Circ Res. 2012;111:863-75.

34. Schneeberger M, Dietrich MO, Sebastian D, Imbernon M, Castano C, Garcia A, et al. Mitofusin 2 in POMC neurons connects ER stress with leptin resistance and energy imbalance. Cell. 2013;155:172-87.

35. Li D, Li X, Guan Y, Guo X. Mitofusin-2-mediated tethering of mitochondria and endoplasmic reticulum promotes cell cycle arrest of vascular smooth muscle cells in G0/G1 phase. Acta Biochim Biophys Sin (Shanghai). 2015;47:441-50.

36. Cosson P, Marchetti A, Ravazzola M, Orci L. Mitofusin-2 independent juxtaposition of endoplasmic reticulum and mitochondria: an ultrastructural study. PLoS ONE 2012;7:e46293.

37. Leal NS, Schreiner B, Pinho CM, Filadi R, Wiehager B, Karlstrom $\mathrm{H}$, et al. Mitofusin-2 knockdown increases ERmitochondria contact and decreases amyloid beta-peptide production. J Cell Mol Med 2016;20:1686-95.

38. Filadi R, Greotti E, Turacchio G, Luini A, Pozzan T, Pizzo P. Presenilin 2 modulates endoplasmic reticulum-mitochondria coupling by tuning the antagonistic effect of Mitofusin 2. Cell Rep.2016;15:2226-38.

39. Zampese E, Fasolato C, Kipanyula MJ, Bortolozzi M, Pozzan T, Pizzo P. Presenilin 2 modulates endoplasmic reticulum (ER)mitochondria interactions and $\mathrm{Ca} 2+$ cross-talk. Proc Natl Acad Sci USA. 2011;108:2777-82. 
40. De Vos KJ, Morotz GM, Stoica R, Tudor EL, Lau KF, Ackerley $\mathrm{S}$, et al. VAPB interacts with the mitochondrial protein PTPIP51 to regulate calcium homeostasis. Hum Mol Genet. 2012;21: $1299-311$.

41. Eiyama A, Okamoto K. PINK1/Parkin-mediated mitophagy in mammalian cells. Curr Opin Cell Biol. 2015;33:95-101.

42. Calì T, Ottolini D, Negro A, Brini M. Enhanced parkin levels favour ER-mitochondria crosstalk and guarantee $\mathrm{Ca}^{2+}$ transfer to sustain cell bioenergetics. Biochim Biophys Acta.2013;1832: 495-508.

43. Zheng L, Bernard-Marissal N, Moullan N, D'Amico D, Auwerx J, Moore DJ, et al. Parkin functionally interacts with PGC-1alpha to preserve mitochondria and protect dopaminergic neurons. Hum Mol Genet. 2017;26:582-98.

44. Gautier CA, Erpapazoglou Z, Mouton-Liger F, Muriel MP, Cormier F, Bigou S, et al. The endoplasmic reticulum-mitochondria interface is perturbed in PARK2 knockout mice and patients with PARK2 mutations. Hum Mol Genet. 2016;25:2972-84.

45. Ryan MD, King AM, Thomas GP, Cleavage of foot-and-mouth disease virus polyprotein is mediated by residues located within a 19 amino acid sequence. J Gen Virol. 1991;72(Pt 11):2727-32.

46. Denison SR, Wang F, Becker NA, Schule B, Kock N, Phillips LA, et al. Alterations in the common fragile site gene Parkin in ovarian and other cancers. Oncogene. 2003;22:8370-8.

47. Pawlyk AC, Giasson BI, Sampathu DM, Perez FA, Lim KL, Dawson VL, et al. Novel monoclonal antibodies demonstrate biochemical variation of brain parkin with age. J Biol Chem. 2003;278:48120-8.

48. Kim JH, Lee SR, Li LH, Park HJ, Park JH, Lee KY, et al. High cleavage efficiency of a $2 \mathrm{~A}$ peptide derived from porcine teschovirus-1 in human cell lines, zebrafish and mice. PLoS ONE. 2011;6:e18556.

49. Bannai $\mathrm{H}$, Inoue $\mathrm{T}$, Nakayama $\mathrm{T}$, Hattori M, Mikoshiba K, Kinesin dependent, rapid, bi-directional transport of ER subcompartment in dendrites of hippocampal neurons. J Cell Sci.2004;117(Pt 2):163-75.

50. Villegas R, Martinez NW, Lillo J, Pihan P, Hernandez D, Twiss $\mathrm{JL}$, et al. Calcium release from intra-axonal endoplasmic reticulum leads to axon degeneration through mitochondrial dysfunction. J Neurosci.2014;34:7179-89.

51. Cui-Wang T, Hanus C, Cui T, Helton T, Bourne J, Watson D, et al. Local zones of endoplasmic reticulum complexity confine cargo in neuronal dendrites. Cell. 2012;148:309-21.
52. Twig G, Elorza A, Molina AJ, Mohamed H, Wikstrom JD, Walzer $\mathrm{G}$, et al. Fission and selective fusion govern mitochondrial segregation and elimination by autophagy. EMBO J. 2008;27:433-46.

53. Sarraf SA, Raman M, Guarani-Pereira V, Sowa ME, Huttlin EL, Gygi SP, et al. Landscape of the PARKIN-dependent ubiquitylome in response to mitochondrial depolarization. Nature. 2013;496:372-6.

54. Spillane M, Ketschek A, Merianda TT, Twiss JL, Gallo G. Mitochondria coordinate sites of axon branching through localized intra-axonal protein synthesis. Cell Rep. 2013;5:1564-75.

55. Tang F, Kalil K. Netrin-1 induces axon branching in developing cortical neurons by frequency-dependent calcium signaling pathways. J Neurosci. 2005;25:6702-15.

56. Bergamin G, Cieri D, Vazza G, Argenton F, Mostacciuolo ML. Zebrafish $\mathrm{Tg}$ (hb9: MTS-Kaede): a new in vivo tool for studying the axonal movement of mitochondria. Biochim Biophys Acta. 2016;1860:1247-55.

57. Pauls S, Zecchin E, Tiso N, Bortolussi M, Argenton F. Function and regulation of zebrafishnkx2.2a during development of pancreatic islet and ducts. Dev Biol. 2007;304:875-90.

58. Ottolini D, Cali T, Brini M. Methods to measure intracellular $\mathrm{Ca}(2$ +) fluxes with organelle-targeted aequorin-based probes. Methods Enzymol. 2014;543:21-45.

59. Pinaud F, Dahan M. Targeting and imaging single biomolecules in living cells by complementation-activated light microscopy with split-fluorescent proteins. Proc Natl Acad Sci USA. 2011;108: E201-10.

60. Giacomello M, Drago I, Bortolozzi M, Scorzeto M, Gianelle A, Pizzo P, et al. $\mathrm{Ca} 2+$ hot spots on the mitochondrial surface are generated by $\mathrm{Ca} 2+$ mobilization from stores, but not by activation of store-operated Ca2 + channels. Mol Cell. 2010;38:280-90.

61. Smirnova E, Shurland DL, Ryazantsev SN, van der Bliek AM. A human dynamin-related protein controls the distribution of mitochondria. J Cell Biol. 1998;143:351-8.

62. Frank S, Gaume B, Bergmann-Leitner ES, Leitner WW, Robert EG, Catez F, et al. The role of dynamin-related protein 1, a mediator of mitochondrial fission, in apoptosis. Dev Cell. 2001;1:515-25.

63. Tokuyasu KT. A technique for ultracryotomy of cell suspensions and tissues. J Cell Biol. 1973;57:551-65.

64. Schindelin J, Arganda-Carreras I, Frise E, Kaynig V, Longair M, Pietzsch T, et al. Fiji: an open-source platform for biologicalimage analysis. Nat Methods. 2012;9:676-82. 